\title{
Complex Intratarsal Cyst with a Mixed Ciliated Respiratory-Type and Squamous Epithelial Lining
}

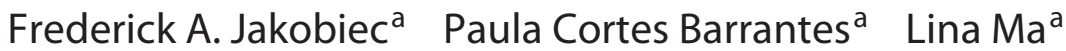 \\ Nahyoung Grace Lee ${ }^{b}$
}

a David G. Cogan Laboratory of Ophthalmic Pathology, Massachusetts Eye and Ear Infirmary/Harvard Medical School, Boston, MA, USA; ${ }^{b}$ Department of Oculoplastics, Massachusetts Eye and Ear Infirmary/Harvard Medical School, Boston, MA, USA

\section{Established Facts}

- Intratarsal cysts are either congenital, acquired, or develop after trauma or prior surgery.

- A cyst's lining governs its diagnosis, and together with its location, helps to determine its origin.

- An intratarsal cyst with a partially ciliated respiratory and coexistent squamous epithelial lining has never been reported before.

\section{Novel Insights}

- The ciliated respiratory epithelium of an intratarsal cyst may represent the persistence of a ciliated stage of epithelium during the embryonic development of adnexal structures.

- The current lesion displayed the histopathologic and immunohistochemical features of ciliated respiratory epithelium that was probably aberrantly thwarted from complete normal squamous differentiation, rather than the consequence of a displacement of a sinus stem cell.

\section{Keywords}

Eyelid · Tarsus · Cyst · Cilia - Respiratory epithelium . Squamous epithelium · Cytokeratins - Intratarsal keratinous cyst

\section{Abstract \\ A 55-year-old woman developed a painless, non-ulcerated left upper eyelid swelling over 6 months. Examination dis- closed a fluctuant mass that permitted movement of the eye- lid skin over the lesion. A full-thickness eyelid resection con-}

tained a well-encapsulated cyst with milky contents that was predominantly located in the tarsus. The cyst's lining was partially composed of segments of ciliated respiratory-type and non-keratinizing squamous epithelia. Immunohistochemical evaluation with cytokeratins 17, 18, and 19 confirmed the staining pattern of a respiratory-type epithelial cell (whether or not cilia were present in the non-squamous epithelial zones). In the squamous region, entirely different cytokeratin results were obtained vis-a-vis the non-squamous regions of the lining. The current lesion is interpreted as congenital and representing an in situ persistence of embryonic ciliated glan-

\section{KARGER}

(c) 2019 S. Karger AG, Basel 
dular epithelium that normally exists only transitorily. A more remote possibility is that the lesion was the result of ectopic epithelial cells displaced from an adjacent sinus. A recurrence has not developed during 6 months of follow-up.

(C) 2019 S. Karger AG, Basel

\section{Introduction}

Respiratory cysts that are not due to congenital anomalies or prior sinus disease, sinus surgery, or sinus trauma are exceptionally rare and must be distinguished from mucoceles encroaching on the orbit [1-4]. They have been described in the medial eyelid and in various precincts of the orbit. In this article an intratarsal cyst of the upper eyelid is described, the lining of which exhibited equal components of both respiratory-type ciliated epithelium with goblet cells and squamous epithelium. Immunostaining demonstrated cytokeratins typical of respiratory epithelium in the non-squamous ciliated and non-ciliated segments of the cyst's lining. These studies also assisted in distinguishing the lesion from an intratarsal keratinous cyst (ITKC), a close clinical simulator $[3,5]$.

\section{Clinical History}

A 55-year-old woman presented with a slowly growing, painless, non-erythematous, and non-ulcerated swelling of the right upper eyelid (because of its unprepossessing and non- distinctive appearance, a clinical photograph was not obtained). It had been present for 6 months and displayed intermittent drainage of milky fluid from the eyelid. The patient gave a history of substance abuse, hepatitis C, HIV, and diabetes mellitus. On clinical examination, the lesion in the right upper eyelid was non-tender to palpation and measured approximately $10 \mathrm{~mm}$ in diameter. It was felt to involve the tarsus. The overlying skin was movable over the lesion. The mass had a rubbery consistency with central fluctuance, suggesting that it was at least partially cystic. When lanced with a needle, it produced a viscous, mucoid, whitish-yellow fluid. Due to the cystic nature and intratarsal location of the lesion, a decision was made to resect it in toto within its capsule via a full-thickness eyelid wedge resection. The specimen was placed in formalin. The eyelid wound was reapproximated with 7-0 and 6-0 vicryl sutures. The surgical site has healed well without a recurrence during 9 months of follow-up.

\section{Results}

\section{Histopathologic Findings}

A full-thickness portion of an upper eyelid contained a cyst with milky luminal contents, most of which had been released during the performance of surgery. The specimen measured $1.3 \times 0.6 \times 0.5 \mathrm{~cm}$ and included skin, eyelid margin with eye lashes, and tarsal conjunctiva (Fig. 1a). The cyst was predominantly located within the tarsus but protruded into the anterior lamellar tissue planes of the eyelid (Fig. 1b, c).

A thick fibrous wall that was continuous with the tarsus enveloped the cyst, which displayed a small intraoperative luminal hemorrhage (Fig. 1c). An inflammatory lipogranulomatous nodule was observed in the anterior-most wall of the cyst and nearby tissues (Fig. 1a, b). The other anatomic structures of the involved eyelid such as the lacrimal accessory glands of Wolfring in the superior poles of the tarsus, the intratarsal Meibomian sebaceous glands, the cilia, the apocrine glands of Moll, and the marginal bundle of the orbicularis muscle (the muscle of Riolan) were all well-formed and intact (Fig. 1d; top and bottom panels).

Two types of epithelial lining were found in the cyst. About $50 \%$ was composed by either a thin lamina of $2-3$ layers of loosely adherent non-squamous cuboidal cells or multilaminar thicker zones of non-squamous epithelium (Fig. 1e, f). In some regions, the apical cytoplasm was prominently eosinophilic with a suggestion of projecting cilia and/or microvilli. Basal cell hyperplasia was the cause of the thicker regions of the cyst's lining (Fig. 1e, f; see bottom cellular layer of lining). In the thicker regions, a pseudostratified arrangement was not in evidence due to poor nuclear registration.

Histochemical staining with phosphotungsic acid hematoxylin revealed the presence of adlumenal cilia exhibiting brush-like bouquets (Fig. 2a, b). Goblet cells were generously dispersed among the non-squamous cells (Fig. 2c; left panels). Where there was basal cell hyperplasia, goblet cells were aggregated on the surface of the nonsquamous epithelium (Fig. 2c; right panel). The other half of the cyst's lining was constituted by thin or thick zones of non-keratinizing squamous epithelium (Fig. 2d) devoid of keratohyaline granules (Fig. 2e, f). Focal areas of the superficial epithelium manifested delamination of string-like keratin without a surface cuticular membrane or keratohyaline granules (Fig. 2f).

\section{Immunohistochemical Findings}

The extra-cystic inflammatory nodule was a foreignbody granuloma (Fig. 3a) with giant cells and mononuclear epithelioid cells that immunostained positively for CD68 and CD163. (Fig. 3b; left panel). Cytokeratin (CK) immunostaining disclosed the engulfment by histiocytes and epithelioid cells of CK 7-, CK 14-, CK 17-, and CK 19-positive keratin flakes (Fig. 3b; right panel). Testing for CK 18 residues was negative. 
Fig. 1. Histologic features of a complex intratarsal cyst. a A full-thickness upper eyelid resection displays an intratarsal cyst (C) with a thin surviving portion of the tarsus (arrow) beneath the tarsal conjunctiva. Normal tarsus $(\mathrm{T})$ is observed on either side of the cyst. A small nodule (crossed arrows) is located near the cyst wall. Hematoxylin and eosin stain (H\&E). b Intact Meibomian glands within the undistorted portions of the tarsus (T). The thick fibrous wall of the cyst is an extension of the dense tarsal collagen (arrows). The nodule near the anterior cyst wall is actually a lipogranuloma (LG). Masson's trichrome stain, $\times 2$. c A small intraoperative hemorrhage is identified in the lumen (L) of the cyst. The arrow indicates the thin lamina of tarsal collagen surviving beneath the conjunctiva. The adjacent tarsus $(\mathrm{T})$ is normal with a clear Meibomian gland. Masson's trichrome stain, $\times 4$. $\mathbf{d}$ Top panel: An accessory lacrimal gland of Wolfring (W) is juxtaposed to a Meibomian gland (M) in the upper pole of the tarsus. $H \& E$ stain, $\times 4$. Bottom panel: An intact muscle of Riolan (RM) is present near the eyelid margin. Masson's trichrome stain, $\times 10$. $e$ In the region of the cyst, the lumen $(\mathrm{L})$ is formed by a thin non-squamous epithelium above and a thicker nonsquamous epithelium below, the latter due to basal cell hyperplasia (arrows). $\mathrm{H} \& \mathrm{E}$ stain, $\times 40$. f Higher power microscopy reveals apical projections (arrows) towards the lumen (L) and more ample adlumenal cytoplasm along with basal cell hyperplasia. H\&E stain, $\times 60$.

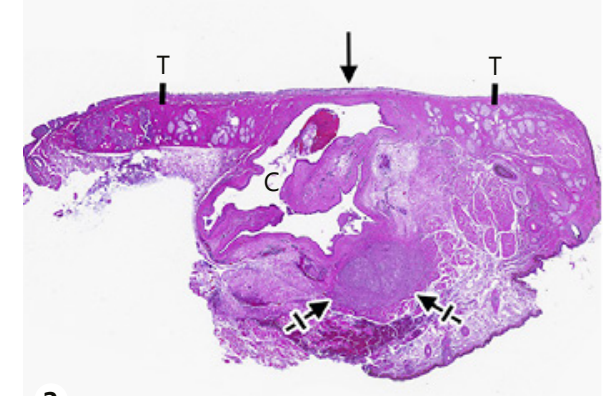

a
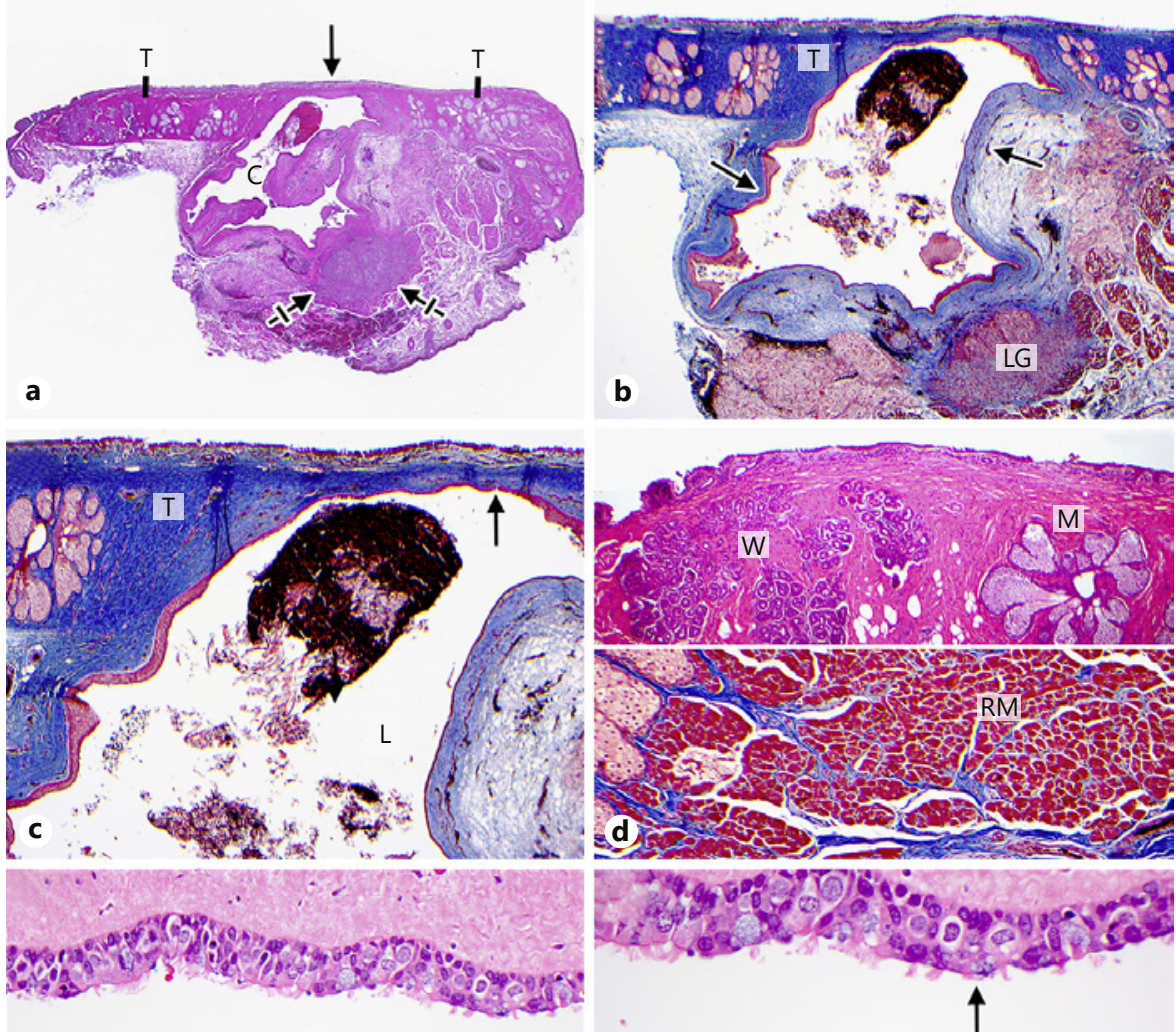

L
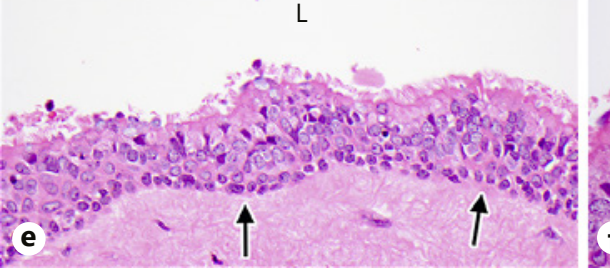

The results of the immunohistochemical staining for $\mathrm{cy}-$ tokeratins of the various segments of the epithelial nonsquamous lining overlapped considerably and contrasted with the squamous lining of ITKC and normal conjunctival epithelium (see Table 1 and Fig. 3c, d, 4). In the following illustrations, the panels on the left and middle are from the zones with thin and thick non-squamous linings; those on the far right represent the squamous lining staining results (for both thin and thick squamous segments). CK $7 \mathrm{immu-}$ nostained the suprabasal cells of the thin non-squamous regions but not the basal cells; the thick non-squamous regions evinced negative staining of the basal cell hyperplasia (Fig. 3c). CK 14 stained the basal cells of the thin and thick non-squamous cellular regions but not the suprabasal or surface cells (Fig. 3d). CK 17 displayed the same staining pattern as CK 14 (Fig. 4a). CK 18 immunostained only the superficial cells of the thin and thick non-squamous segments (Fig. 4b). Finally, CK 19 stained the full thickness of the non-squamous thin epithelium but only the superficial cells of the thick non-squamous zones, sparing the hyperplastic basal cells (Fig. 4c). The squamous regions were CK 7 (Fig. 3c, right panel) and CK 18 (Fig. 4c; right panel) totally negative, while CK 14 (Fig. 3d; right panel) and CK 17 (Fig. 4a; right panel) were full thickness intensely positive. CK 19 was only faintly and spottily positive (Fig. 4c; right panel). There was no overlap or transitioning between the cytokeratin staining patterns or the non-squamous and squamous segments of the cyst's lining.

\section{Discussion}

From the clinical perspective, the cyst reported herein had 2 characteristics mimicking ITKC [6]: its upper eyelid location beneath the dermis and orbicularis muscle and these tissues' free mobility over the mass. When the 

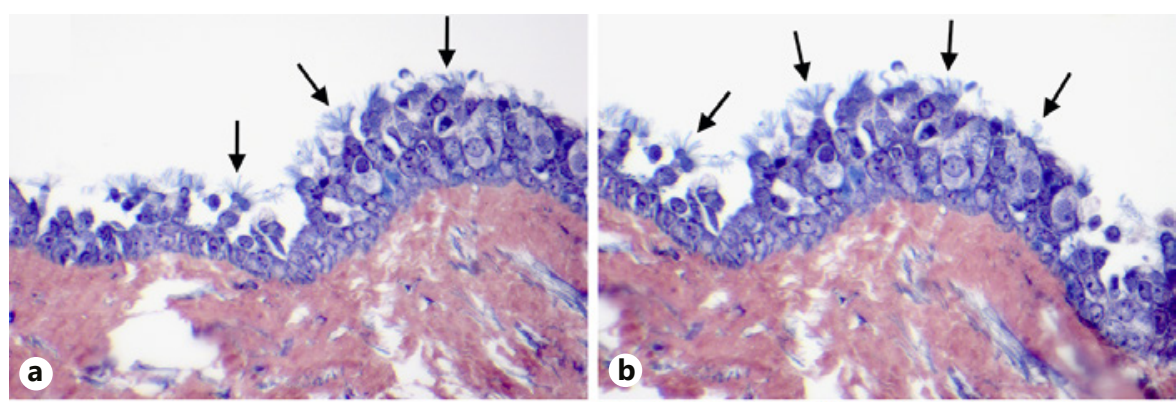

Fig. 2. Histochemical staining of non-squamous and squamous segments of lining. a, b Two photomicrographs of phosphotungstic acid hematoxylin staining at slightly different focuses. Note the bouquets of projecting apical cilia (arrows). Phosphotungstic acid and hematoxylin stainPTAH, $\times 60$. c Left panel: Single Alcian blue-positive goblet cells in the thin nonsquamous epithelium. Right panel: Surface clusters of goblet cells surmounting an area of focal basal cell hyperplasia (BCH). Alcian blue stain, $\times 60$. $\mathbf{d}$ Thin (top) and thick (bottom) squamous epithelium delimits the cyst's lumen (L). H\&E stain, $\times 20$. e The multilaminar non-keratinizing squamous epithelium lacks a keratohyaline granular layer or a surface cuticle towards the lumen (L). H\&E stain, $\times 40$. $f$ A rare focus of delamination of stringy strands of keratin $(\mathrm{K})$ produced by the squamous lining in the absence of keratohyaline layer. H\&E stain, $\times 40$.

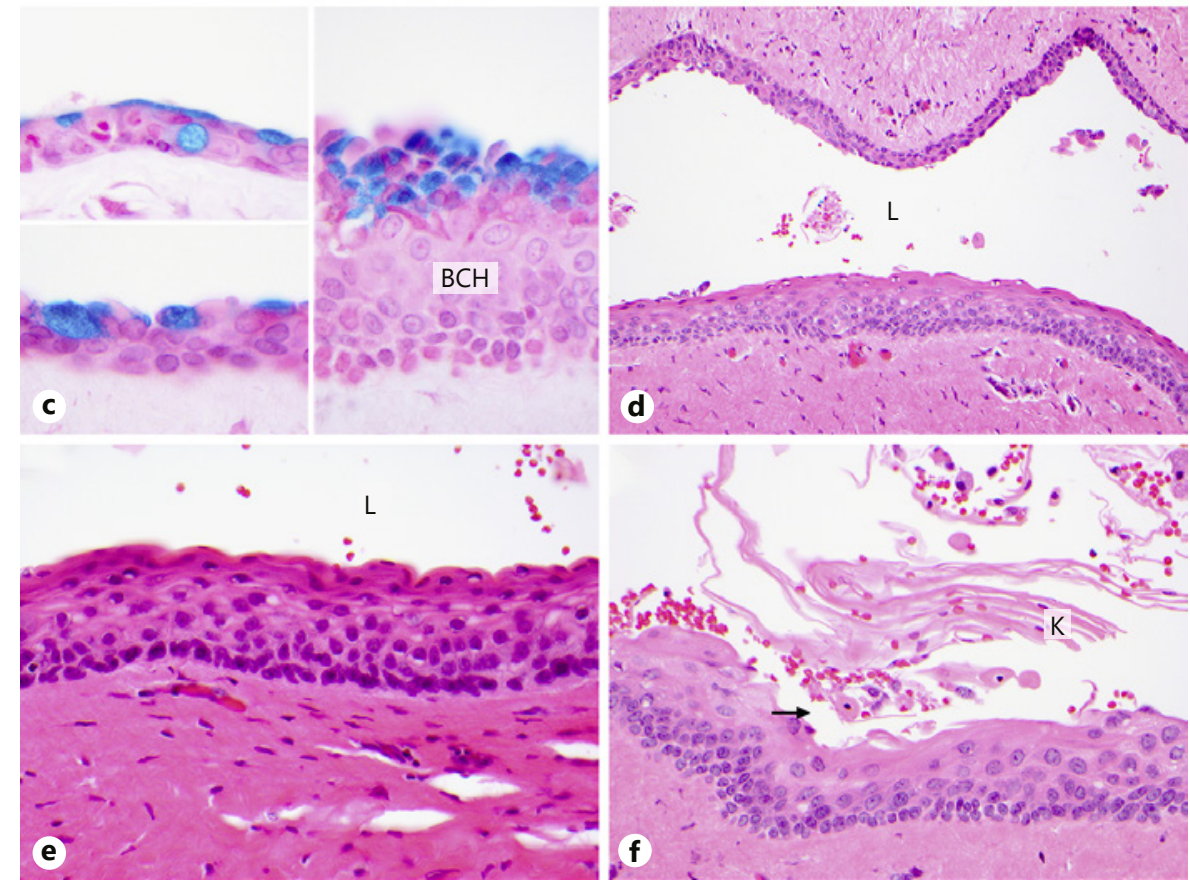

cyst was examined histopathologically, however, it displayed stark differences from an ITKC.

Most conspicuous was the absence of a surface cuticular membrane from the squamous regions of the epithelial lining, which is typically observed in ITKC [6]. Furthermore, the contents of the current cyst were milky and viscous rather than the solid luminal keratinous contents of an ITKC. The lining of the current cyst was composed of non-keratinizing squamous epithelium alternating with a ciliated or non-ciliated respiratory-type epithelium [3-5] (Table 1). We have employed the term "respiratory-type" epithelium because we do not believe the lesion stemmed from a displacement of cells from an adjacent sinus (see below). The non-squamous ciliated and non-ciliated epithelial portions accounted for approximately $50 \%$ of the lining, while the squamous cells were responsible for the other $50 \%$. The squamous component focally shed linear strands of refractile keratin into the lumen, thereby demonstrating its capacity for keratiniza- tion without, however, detectable keratohyaline granules. There were widely scattered goblet cells within the respiratory zones that were highlighted with Alcian blue, a feature not evinced by the squamous lining in the current lesion or in an ITKC. Phosphotungsic acid hematoxylin staining verified the presence of apical cilia only in the respiratory-type epithelial regions.

The ciliated epithelium ranged from 3 to 6 cells in thickness, the thicker areas reflecting basal and suprabasal cell hyperplasia. Distinctive surface cells with copious apical eosinophilic cytoplasm were also seen. The nuclei of the non-squamous cells failed to manifest a regimented pseudostratified arrangement in the zones with basal and/or suprabasal cell hyperplasia [5]; the non-ciliated epithelium was 2-3 cells in thickness with dispersed goblet cells and other scattered cells exhibiting perinuclear haloes (possibly goblet cells that had discharged their mucus). The squamous cells for the most part were multilaminar and non-keratinizing; they displayed clear-cut 
Fig. 3. Granuloma and results of immunohistochemical staining. a A granuloma with histiocytes, xanthoma cells, and lymphocytic infiltration constitutes the nodule identified adjacent to a leak in the cyst's wall. $H \& E$ stain, $\times 20$. b Left panel: CD 68-positive histiocytes are abundantly present in the granuloma. Notice the multinucleated giant cell (arrow). Immunoperoxidase with diaminobenzidine-DAB chromogen and hematoxylin counterstain, $\times 20$. The inset depicts a CD 163-positive multinucleated giant cell. Immunoperoxidase stain, $\times 60$. Right panel: CK 19 -positive flakes of keratin debris are found in the histiocytes of the granuloma. Immunoperoxidase stain, $\times 10$. c Left panel: CK 7 positively stains the suprabasal cells in the thin non-squamous epithelium. The basal cells are negative (arrows). Center panel: CK stains the suprabasal cells in the thick nonsquamous epithelium but not the hyperplastic base cells (arrow). Right panel: CK 7 is negative in the squamous epithelium. Immunoperoxidase stain, $\times 60$. d Left panel: CK 14 stains only the basal cells in the thin non-squamous epithelium (arrow). Center panel: The hyperplastic basal cells are positive for CK 14 (arrow). Right panel: The squamous epithelium is full thickness CK 14 positive. Immunoperoxidase stain, $\times 60$.
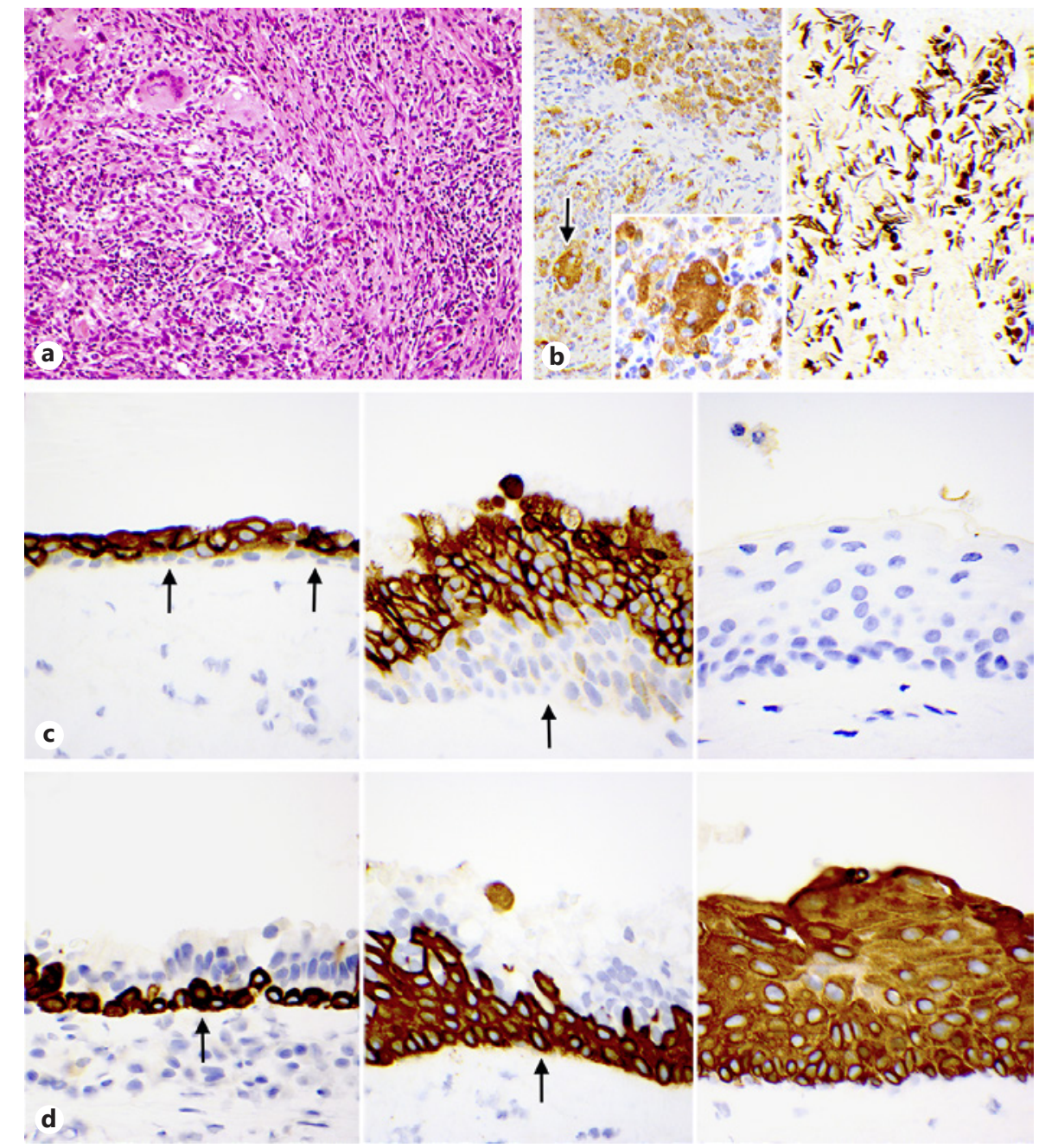

cell borders and occasional focal areas of string-like keratinization without keratohyaline granules in the superficial cells.

Immunohistochemically, the ciliated epithelium stained like true respiratory epithelium of the sinuses (Table 1). CK 17 and 18 were positive (in contrast to CK 17 negativity in the conjunctival epithelium). The nonciliated/non-squamous epithelium manifested the same staining results, thereby establishing a close affinity between the non-squamous ciliated and non-ciliated segments. The absence of cilia was probably a secondary degenerative phenomenon in many regions of the cyst's non-squamous lining. The squamous portions of the present cyst's lining immunostained with altogether different results (Table 1); for example, CK 14 and 17 were full thickness positive, but CK 7 was negative. The squamous segments could represent a metaplasia of the respiratory epithelium from chronic irritation or else the nor- mal embryologic transformation of the ephemeral ciliated cells into squamous cells. These segments did not display the expected staining properties of a Meibomian gland duct from which an ITKC generates [6] (Table 1).

We believe that the current cyst was congenital in origin. It had expanded in the region of the mid-tarsus creating its near dissolution; only a thin band of dense subconjunctival tarsal collagen bridged the upper and lower halves of the intact and uninvolved portion of the tarsus. The tarsus was otherwise well developed with normal Wolfring glands, Meibomian glands, Moll's apocrine glands, intact cilia, and well-developed bundles of Riolan's muscle in the subepithelial eyelid margin. The cyst expanded outwards into the pretarsal anterior eyelid tissues where the tarsal collagenous wall had become thinner. The densely collagenized fibrous wall supporting the cyst was actually constituted by the tarsal collagen, as demonstrated with the Masson trichrome stain that vividly highlighted that the cystic wall's thick col- 
Fig. 4. Additional cytokeratin immunohistochemical staining results. a Left panel: CK 17 is positive in the basal cells of the thin non-squamous epithelium. Center panel: The hyperplastic non-squamous basal cells are CK 17 positive (arrow). Right panel: The squamous epithelium is full thickness CK 17 positive. Immunoperoxidase stain, $\times 60$. b Left panel: CK 18 only stains the superficial non-squamous cells in a thin epithelial segment. Center panel: CK 18 stains at least two cell layers of the superficial cells in the thick non-squamous epithelium. The hyperplastic non-squamous basal cells are negative (arrow). Right panel: CK 18 is completely negative in the squamous epithelium. Immunoperoxidase stain, $\times 60$. c Left panel: CK 19 stains all of the cells in the thin non-squamous epithelium. Center panel: Only the most superficial cells stain positively for CK 19 in the thick non-squamous portion of the epithelial lining. Right panel: Faint and for the most part spotty staining of the squamous epithelium for CK 19 (arrow). Immunoperoxidase stain, $\times 60$.
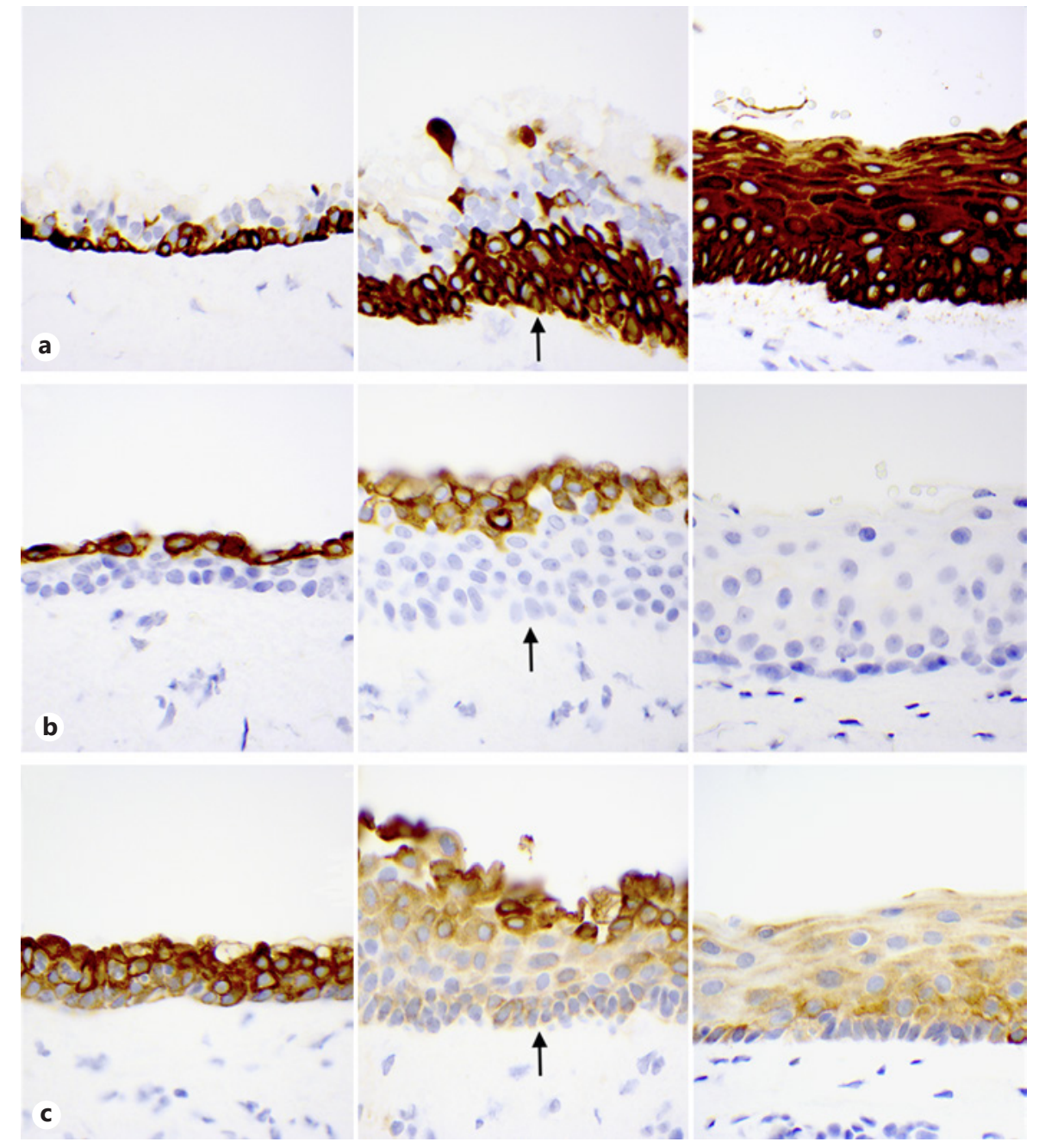

lagen fibers derived in continuity from the tarsus. A focal rupture in the thinnest region of the anterior wall of the cyst was responsible for the adjacent lipogranuloma with entrapped keratin fragments, as demonstrated with cytokeratin immunostaining (only CK 18 was not detected).

How is it possible that respiratory-type ciliated epithelial could be found in the tarsus? One can only speculate on this question. A wide variety of cysts can occur in the orbit [7], including some with a ciliated epithelium. Ciliated cells have been observed in cutaneous adnexal ducts ephemerally during their embryologic development [8] as well as in odontogenic keratocysts [9]. An elongated tubular structure with ciliated luminal cells has been noted adjacent to an ectopic tooth lodged in a congenital cyst of the lower eyelid [10], presumably arising from an ectopia of embryonic buccal epithelium that had been displaced laterally during morphogenesis of the cephalic region. The literature contains other reports of orbital cysts containing an abortive tooth structure in a lumen delimited by ciliated epithelium [10]. The embryonic buccal mucosa is also temporarily ciliated [11]. Our favored conjecture is that the present lesion represents a portion of ciliated embryonic eyelid glandular epithelium that endured in situ from the embryonic period throughout later life. Part of the cyst's lining was somehow able to sustain a ciliated respiratory-type epithelial expression over a long period of the patient's life. Other parts of the embryonic epithelium in the cyst managed to differentiate into normal mature squamous epithelium. The nonsquamous/non-ciliated epithelium of the cyst's lining had a cytokeratin staining pattern identical to that of sinus ciliated epithelium and was probably a partially degenerate, but still viable, form of respiratory epithelium.

The foregoing findings suggest that the cytokeratin profile of respiratory-type epithelium is a reflection of an arrest in cytoarchitectural maturation of an embryonic 


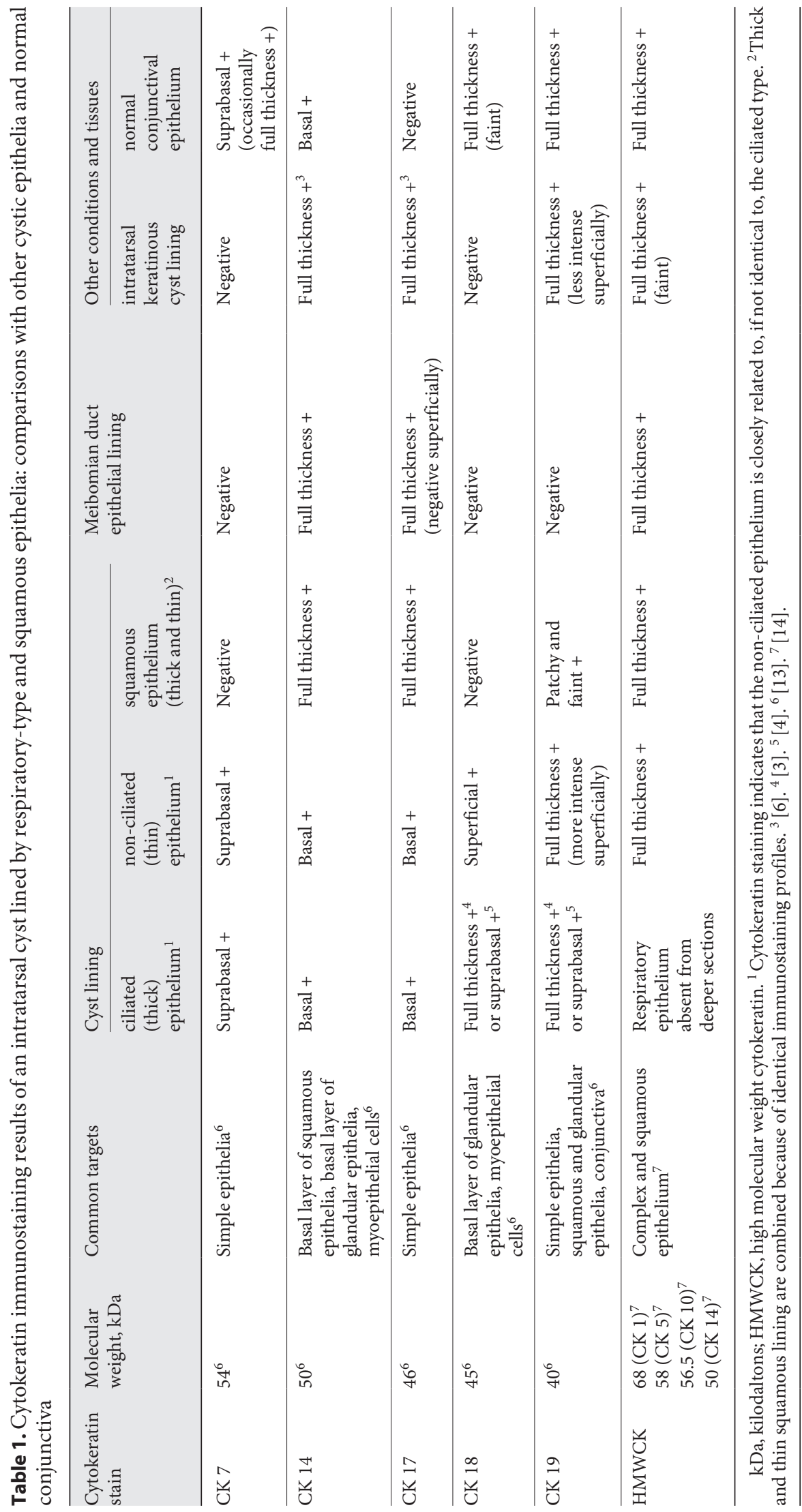


epithelium rather than a sign of its precise provenance or initial anatomic location. The concepts of an ectopia of genuine embryonic respiratory cells displaced into the tarsus from an adjacent sinus or from the buccal mucosa during embryogenesis seem less plausible to us. On the other hand, an example of a true choristomatous displacement of tissue into the orbit is an enterogenous cyst arising from embryonic cells displaced from the most rostral portion of the foregut $[7,12]$.

\section{Statement of Ethics}

This case report complies with the tenets of the Declaration of Helsinki and Health Insurance Portability and Accountability Act Regulations.

\section{Disclosure Statement}

The authors have no conflicts of interest to declare.

\section{Funding Sources}

Department of Ophthalmology Research Fund, Massachusetts Eye and Ear Infirmary.

\section{Author Contributions}

F.A.J.: main author, responsible for supervision, conceptual guidance, and writing of the manuscript. P.C.B.: responsible of data summary, preparation, library search, and references. L.M.: responsible for illustrations. N.G.L.: provided the case report.

\section{References}

1 Mee JJ, McNab AA, McKelvie P. Respiratory epithelial orbital cysts. Clin Exp Ophthalmol. 2002 Oct; 30(5):356-60.

2 Newton C, Dutton JJ, Klintworth GK. A respiratory epithelial choristomatous cyst of the orbit. Ophthalmology. 1985 Dec;92(12): 1754-7.

3 Thanos A, Jakobiec FA, Mendoza PR, Hatton MP. Ectopic (choristomatous) orbital respiratory cyst: histopathology and immunohistochemistry. Surv Ophthalmol. 2014 May-Jun; 59(3):328-33.

4 Jakobiec FA, Roh M, Stagner AM, Yoon MK. Choristomatous respiratory cyst restricted to the upper eyelid. Ophthal Plast Reconstr Surg. 2016 Jan-Feb;32(1):e15-8.

5 Michael H. Ross, Wojciech Pawlina. Histology: A text and atlas with correlated cell and molecular biology. Ed 6. Wolters Kluwer Health/ Lippincott Williams and Wilkins. 2001. Chapter 19. pp. 664-87.
6 Jakobiec FA, Mehta M, Iwamoto M, Hatton MP, Thakker M, Fay A. Intratarsal keratinous cysts of the Meibomian gland: distinctive clinicopathologic and immunohistochemical features in 6 cases. Am J Ophthalmol. 2010 Jan; 149(1):82-94.

7 Jakobiec FA, Bhat P, Kropp TM. Palpebroorbital apocrine cystadenoma: immunohistochemical verification of a unique variant with a critical differential diagnosis. Ophthal Plast Reconstr Surg. 2010 Jul-Aug;26(4):245-9.

8 Hashimoto K, Gross BG, Lever WF. The ultrastructure of human embryo skin. II. The formation of intradermal portion of the eccrine sweat duct and of the secretory segment during the first half of embryonic life. J Invest Dermatol. 1966 Jun;46(6):513-29.

9 Piecuch JF, Eisenberg E, Segal D, Carlson R. Respiratory epithelium as an integral part of an odontogenic keratocyst: report of case. J Oral Surg. 1980 Jun;38(6):445-7.
10 Jakobiec FA, Nguyen J, Mandell K, Fay A. Complex palpebral odontogenic choristoma: A reappraisal of the origin of teeth-bearing periocular lesions. Am J Ophthalmol. 2009 Mar;147(3):531-543.e1.

11 Zarbo R, Torres F, Gomez J. Nasal cavity and paranasal sinuses. Embryology, anatomy, histology and pathology. Head and neck surgical pathology. In: Pilch B, editor. Philadelphia: Lippincott, Williams and Wilkins; 2001. p. 106.

12 Leventer DB, Merriam JC, Defendini R, Behrens MM, Housepian EM, LeQuerica S, et al. Enterogenous cyst of the orbital apex and superior orbital fissure. Ophthalmology. 1994 Sep;101(9):1614-21.

13 Dabbs DJ. Diagnostic Immunohistochemistry: Theranostic and genomic implications. Elsevier, 4th Edition. 2014. Philadelphia, PA. p. 212.

14 Enzo Biochem Inc. Datasheet for cytokeratin $1 / 5 / 10 / 14$ human monoclonal antibody (34BE12). 